\section{A importância do Serviço de Informações sobre Agentes Teratogênicos, Bahia, Brasil, na prevenção de malformações congênitas: análise dos quatro primeiros anos de funcionamento}

\author{
Importance of the Teratogen Information Service \\ in Bahia, Brazil, for prevention of congenital \\ malformations: an initial four-year review
}

\author{
${ }_{1}^{1}$ Faculdade de Medicina da \\ Bahia, Universidade Federal \\ da Bahia, Salvador, Brasil. \\ 2 Instituto de Biologia, \\ Universidade Federal da \\ Bahia, Salvador, Brasil. \\ Correspondência \\ M. B. Toralles \\ Departamento de Pediatria, \\ Faculdade de Medicina da \\ Bahia, Universidade Federal \\ da Bahia \\ Rua Waldemar Facão \\ 870/701-B, Salvador, BA \\ 40296-700, Brasil. \\ m.toralles@uol.com.br
}

\begin{abstract}
Teratogenic agents are defined as physical, chemical, or biological agents or nutrient deficiencies that lead to fetal structural or functional alterations. Information on the effects of exposure to teratogens during pregnancy is of the utmost importance. In order to achieve this goal, in 2001, the Bahia State Teratogen Information Service was created in the Medical Genetics Department at the University Hospital of the Federal University in Bahia. The current paper aimed to describe the first four years of operation in the service. From March 2001 to May 2005, the service was consulted by telephone, fax, and e-mail. During this period, 408 queries were made, for a total of 1,091 different reasons. Most queries were made by pregnant women and health care professionals. Hair products, herbal teas, and misoprostol were the most widely investigated agents. The low number of queries (average 1/day) shows the need for more awareness-raising on the risks posed by the various agents.
\end{abstract}

Teratogens; Congenital Abnormalities; Pregnancy; Information Services

\author{
Maria Betânia Toralles 1 \\ Bruno Meira Castro Trindade 1 \\ Luiza Cavalcanti Fadul 1 \\ Clóvis F. Peixoto Junior 2 \\ Marco Antonio Costa Campos de Santana 1 \\ Crésio Alves 1
}

\section{Introdução}

Agente teratogênico é definido como qualquer substância, organismo, agente físico ou estado de deficiência que, estando presente durante a vida embrionária ou fetal, produz alteração na estrutura ou função da descendência 1,2,3,4. Para Hansen et al. ${ }^{2}$, são considerados teratogênicos: (1) agentes que tenham exposição danosa documentada em um determinado período do desenvolvimento pré-natal; (2) substâncias cujos efeitos teratogênicos tenham sido claramente demonstrados através de dados estatisticamente significantes em dois ou mais estudos epidemiológicos; e (3) drogas que, mesmo em rara exposição ambiental, estejam associadas com ocorrência de defeitos congênitos, contanto que exista uma cuidadosa descrição de casos clínicos.

A ação de um agente teratogênico na reprodução humana é variada. Eles podem provocar aborto, malformações, retardo do crescimento intra-uterino ou deficiência mental. Sua ação depende de fatores como: estágio do desenvolvimento do concepto, relação dose-efeito, genótipo materno-fetal e mecanismo patogênico específico de cada agente 5 .

A partir da década de 1980, serviços especializados em informação sobre agentes teratogênicos foram criados em diversos países da Europa e na América do Norte 6,7,8. Esses serviços representam importantes fontes de dados para investigação sobre o potencial teratogênico de 
diversos agentes permitindo conhecer melhor a farmacocinética de várias substâncias 7,8.

No Brasil, o primeiro Serviço de Informação sobre Agentes Teratogênicos (SIAT) foi implantado no Setor de Genética Médica do Hospital das Clínicas de Porto Alegre, Estado do Rio Grande do Sul, em 1990, e vinculado ao Estudo Colaborativo Latino-americano de Malformações Congênitas (ECLAMC) 9. Atualmente, o SIAT funciona também nas cidades do Rio de Janeiro, Salvador (Bahia), Campinas (São Paulo), Fortaleza e Juazeiro do Norte (Ceará) 10 .

Na Bahia, desde sua implantação em março de 2001, no Serviço de Genética Médica do Hospital Universitário Professor Edgard Santos da Universidade Federal da Bahia, o SIAT passou a ser referência para as regiões Norte e Nordeste do país, com atendimento gratuito à população e a profissionais de saúde. As informações colhidas são arquivadas em um banco de dados, proporcionando o desenvolvimento de pesquisas na área de teratogenicidade, reprodução humana e programas de prevenção de anomalias congênitas.

O presente trabalho tem como objetivos: descrever as características do atendimento do SIAT-BA nos quatro primeiros anos de funcionamento, avaliando o perfil dos motivos de consulta e as características da população que utilizou o serviço; e divulgar, para a comunidade, a importância do SIAT na prevenção de malformações congênitas.

\section{Material e métodos}

As consultas foram realizadas por contato via telefone, fax, $e$-mail ou presencialmente, entre março de 2001 e maio de 2005. Para cada consulta, foi preenchida ficha padrão com motivo principal, tipo e período de exposição ao agente, e dados referentes à gestante como: ocupação; idade materna; idade gestacional; trimestre da gestação em que foi realizada a consulta; antecedentes obstétricos; antecedentes de malformações na família; doença crônica pregressa e acompanhamento pré-natal. O uso de cigarros, drogas ilícitas ou álcool também foi registrado.

Após o preenchimento do protocolo, as consultas foram cadastradas no banco de dados desenvolvido em Microsoft Access (Microsoft Corp., Estados Unidos). As pesquisas para resposta às consultas foram realizadas em fontes computadorizadas - TERIS (Teratogen Information System/Shepard's Catalog of Teratogenic Agents; http://depts.washington.edu/terisweb/teris/) e REPROTOX (http://www.reprotox.org) -, assim como em livros e periódicos referentes ao assun- to. No prazo de até 72 horas, a resposta era fornecida via telefone, se o consulente fosse a gestante, e via fax ou $e$-mail, quando o consulente era médico ou outro profissional da área de saúde.

No momento da coleta de dados, esclarecimentos sobre a importância do seguimento foram ressaltados, sendo esse realizado no período de até trinta dias após o parto e com um ano e dois anos, sempre que possível.

\section{Resultados}

O SIAT-BA recebeu, no referido período, 408 consultas, totalizando 1.091 motivos de consulta. A via de consulta mais freqüente foi a telefônica, representando $343(84,1 \%)$ dos casos. Outras vias de consulta foram: $\operatorname{fax}(\mathrm{n}=31 ; 7,6 \%), e$-mail $(\mathrm{n}=8 ; 1,9 \%)$ e presencial $(\mathrm{n}=26 ; 6,4 \%)$.

Identificou-se que 228 (55,9\%) consultas foram realizadas por razão de gestação, enquanto que $117(28,7 \%)$ foram feitas por profissionais de saúde e estudantes, a título de pesquisa. A intenção de engravidar ( $\mathrm{n}=28 ; 6,9 \%$ ), histórico de malformações em gestações passadas $(n=25$; $6,1 \%$ ) e uso de drogas durante a amamentação ( $\mathrm{n}=10 ; 2,4 \%)$ também figuraram entre as principais razões de consulta.

\section{Consulentes}

A maior procura pelo serviço foi realizada pela própria gestante em 171 (42\%) consultas, seguida de médicos e outros profissionais da área de saúde ( $\mathrm{n}=140 ; 34,3 \%)$, além de familiares ( $\mathrm{n}=$ $31 ; 7,6 \%$ ). Nutrizes e mulheres que planejavam engravidar ou com história de aborto, malformações em gestação passada e/ou na família totalizaram $42(10,3 \%)$ consultas, e os estudantes da área de saúde, 20 (4,9\%). Houve perda de informação em apenas 4 (1\%) das fichas.

Em relação à procedência da população assistida, $328(80,4 \%)$ dos consulentes provieram de Salvador, e 49 (12\%), da Grande Salvador e interior da Bahia. Outras localidades totalizaram 26 $(6,4 \%)$ consultas. Com relação à escolaridade, 4 pessoas (1\%) haviam concluído apenas o Ensino Fundamental; 76 (18,6\%), o Ensino Médio; e 237 (58,1\%), o Ensino Superior (Tabelas 1 e 2).

A média de idade das gestantes foi de 29,4 anos (17-47anos). Das 228 gestantes, 115 (50,4\%) encontravam-se na primeira gestação, e 113 (49,6\%) já haviam tido duas ou mais gestações.

No momento da consulta, $125(54,9 \%)$ das gestantes encontravam-se no 1o trimestre, 62 $(27,2 \%)$, no 2 o trimestre, e 33 ( $14,5 \%)$, no 3 o trimestre. História de aborto espontâneo prévio foi relatada por $46(20,2 \%)$ gestantes, sendo que 31 
Procedência dos consulentes do Serviço de Informação sobre Agentes Teratogênicos, Bahia, Brasil (SIAT-BA), 2001-2005.

\begin{tabular}{lcc}
\hline Local & $\mathbf{n}$ & $\%$ \\
\hline Salvador & 328 & 80,4 \\
Grande Salvador & 20 & 4,9 \\
Bahia & 29 & 7,1 \\
Região Nordeste & 4 & 1,0 \\
Região Centro-Oeste & 6 & 1,5 \\
Região Norte & 1 & 0,2 \\
Região Sudeste & 8 & 2,0 \\
Região Sul & 5 & 1,2 \\
Exterior & 2 & 0,5 \\
Sem informação & 5 & 1,2 \\
Total & 408 & 100,0
\end{tabular}

Tabela 2

Escolaridade dos consulentes do Serviço de Informação sobre Agentes Teratogênicos, Bahia, Brasil (SIAT-BA), $2001-2005$.

\begin{tabular}{|c|c|c|}
\hline Escolaridade & $\mathbf{n}$ & $\%$ \\
\hline Analfabeto & 0 & 0,0 \\
\hline \multicolumn{3}{|c|}{ Ensino Fundamental } \\
\hline Incompleto & 6 & 1,5 \\
\hline Completo & 4 & 1,0 \\
\hline \multicolumn{3}{|l|}{ Ensino Médio } \\
\hline Incompleto & 17 & 4,2 \\
\hline Completo & 76 & 18,6 \\
\hline \multicolumn{3}{|l|}{ Ensino Superior } \\
\hline Incompleto & 52 & 12,7 \\
\hline Completo & 237 & 58,1 \\
\hline Sem informação & 16 & 3,9 \\
\hline Total & 408 & 100,0 \\
\hline
\end{tabular}

$(13,6 \%)$ delas apresentavam história anterior de malformação na família.

Dentre as 67 gestantes que consultaram o serviço antes da exposição, 38 (56,7\%) planejaram a gestação, e 28 (41,8\%) não a planejaram. Das 161 gestantes que consultaram o serviço depois de se expor ao motivo da consulta, $111(68,9 \%)$ não planejaram a gestação, e 50 (31\%) a planejaram.

\section{Motivos de consulta}

Dos 1.091 motivos, $615(56,4 \%)$ foram consultados por mulheres em curso de gestação. Desse total, 127 (20,7\%) dos motivos foram consultados sem exposição prévia, enquanto que 488 (79,3\%) foram consultados após exposição (Tabela 3). A média de motivos por consulta foi de 2,67.

As substâncias e agentes mais investigados foram: produtos para cabelo $(n=100 ; 9,2 \%)$, chás ( $\mathrm{n}=44 ; 4 \%)$, misoprostol $(\mathrm{n}=21 ; 1,9 \%)$ e vacina contra rubéola $(\mathrm{n}=21 ; 1,9 \%)$. A Tabela 4 mostra que a maioria das gestantes utilizou as substâncias por indicação médica.

\section{Discussão}

O SIAT-BA funciona há quatro anos, e, nesse período, ocorreram apenas 408 consultas, o que reflete um número ainda muito baixo, tendo em 
Motivos em relação à exposição.

\begin{tabular}{lcc}
\hline Exposição & $\mathbf{n}$ & $\%$ \\
\hline Consultas sem exposição na gestação & 127 & 11,6 \\
Consultas com exposição na gestação & 488 & 44,7 \\
Consultas com exposição pré-concepcional & 85 & 7,8 \\
Consultas durante a amamentação & 13 & 1,2 \\
Consultas devido à exposição paterna & 14 & 1,3 \\
Consultas para fins de pesquisa & 284 & 26,0 \\
Sem informação & 80 & 7,3 \\
Total & 1.091 & 100,0 \\
\hline
\end{tabular}

Tabela 4

Fonte de indicação de uso das substâncias consultadas no Serviço de Informação sobre Agentes Teratogênicos, Bahia, Brasil (SIAT-BA), 2001-2005.

\begin{tabular}{lcc}
\hline Indicação de uso & $\mathbf{n}$ & $\%$ \\
\hline Indicação médica & 338 & 31,0 \\
Sem informação & 187 & 17,1 \\
Não prescrito & 160 & 14,7 \\
Automedicação & 118 & 10,8 \\
Exposição ocupacional & 57 & 5,2 \\
Outros & 231 & 21,2 \\
Total & 1.091 & 100,0
\end{tabular}

vista as 4.504.462 mulheres em idade reprodutiva do Estado da Bahia (Departamento de Informática do SUS; http://w3.datasus.gov.br/datasus/ datasus.php).Há, portanto, necessidade de maior divulgação para a comunidade com relação ao funcionamento do serviço e às vias de consulta acessíveis à população.

Os resultados revelaram que $58,1 \%$ dos consulentes haviam completado o Ensino Superior, ao passo que $1 \%$ concluiu apenas o Ensino Fundamental, sugerindo que, quanto maior o nível de escolaridade, maior a preocupação e procura pelo serviço de orientação. De acordo com censo realizado pelo Instituto Brasileiro de Geografia e Estatística (IBGE; http://www.ibge.gov.br), no Estado da Bahia, em 1996, apenas 2,43\% das pessoas acima de vinte anos de idade apresentavam o Ensino Superior completo. Esse dado demonstra que a população que consulta o SIAT-BA não reflete a realidade local. Verificou-se que, quanto menor o grau de instrução da população, menor é também a preocupação acerca dos possíveis efeitos teratogênicos dos agentes a que são expostas e/ou maior a falta de conhecimento sobre a disponibilidade de consultas ao SIAT.
Dentre os agentes consultados, o misoprostol foi a terceira substância mais pesquisada, sendo que $66,7 \%$ dessas pesquisas estiveram relacionadas ao abortamento. Acredita-se que a facilidade na obtenção de medicamentos e a proibição legal de realizar abortos contribuem para que algumas gestantes lancem mão de métodos abortivos inadequados. Dessa forma, expõem-se a uma grande diversidade de agentes como o misoprostol que, embora não seja um abortivo eficaz, eleva os riscos de anomalias congênitas 11,12.

A vacina contra rubéola ocupou o quarto lugar na lista dos motivos mais pesquisados. Esses dados foram devidos à campanha de vacinação contra rubéola para mulheres em idade fértil, ocorrida no Estado da Bahia, em 2002.

Um número significativo de consultas foi realizado por profissionais de saúde e estudantes a título de pesquisa, o que mostra que o SIAT serve também de apoio a esses profissionais.

As gestantes corresponderam a $42 \%$ dos consulentes, e 183 (80,3\%) dessas consultaram o SIAT após a exposição a um possível agente teratogênico. De acordo com o Estudo Colaborativo Perinatal, $90 \%$ das gestantes utilizam algu- 
ma medicação durante o período gestacional 13 . Esses dados são preocupantes e evidenciam a necessidade de maior esclarecimento para a população de mulheres em idade fértil, a fim de diminuir a exposição a agentes ambientais teratogênicos. É importante que mulheres em idade fértil e gestantes sejam informadas sobre os riscos teratogênicos de exposições a diversas drogas para que essas sejam utilizadas em doses seguras, caso exista documentação na literatura comprovando o seu efeito teratogênico.

Apesar de 80,4\% das consultas terem sido originadas de Salvador, foi interessante constatar que, apesar de ser um serviço local, o atendimento do SIAT não se restringe ao estado, visto que $6,4 \%$ das consultas foram provenientes de outras localidades, pela facilidade de consulta via Internet (e-mail). A divulgação do SIAT é realizada através da distribuição de folhetos explicativos na Secretaria de Saúde do Estado da Bahia, da divulgação pela imprensa e pela veiculação em jornal da Sociedade Regional de Ginecologia. Porém, uma maior divulgação sobre o serviço no interior do Estado da Bahia pode ser de grande ajuda para consulentes gestantes que, em muitas localidades, não têm acesso a um sistema de saúde especializado.

Ao mesmo tempo em que uma boa proporção de mulheres consultou no 1o trimestre, observouse também que a consulta ocorreu somente após a exposição. Esse dado corrobora a necessidade de um trabalho de conscientização por meio de educação continuada da população.
Cerca de 50\% das mulheres que consultaram o SIAT estavam em curso de sua primeira gestação. Essa é uma boa indicação de preocupação, que provavelmente se refletirá nas gestações seguintes, ajudando a prevenir a incidência de malformações.

\section{Conclusão}

O SIAT é um serviço de informação e, de acordo com o número de consultas registradas nestes quatro anos de funcionamento, pôde-se observar que ele é subutilizado em relação ao seu grau de importância preventiva. Faz-se necessária uma maior divulgação do serviço e de sua importância para a população, em especial para as comunidades mais carentes, que, na maioria das vezes, não têm acesso a acompanhamento médico de qualidade.

Os resultados obtidos mostram que há necessidade de informar a população sobre a importância do planejamento da gestação e sobre os riscos oferecidos pelos diversos agentes e, dessa forma, difundir o serviço e incentivar a realização de consultas, principalmente antes da exposição a substâncias, para que a informação concedida aos consulentes possa atuar de forma preventiva.

No momento em que o Ministério da Saúde enfatiza a introdução da Genética Clínica no Sistema Único de Saúde (SUS), o SIAT apresenta-se como importante instrumento de informação na perspectiva da prevenção em nível primário e secundário de malformações congênitas.

\section{Resumo}

Agente teratogênico é definido como o agente físico, químico, biológico ou estado de deficiência que, durante a vida embrionária, leva a alteração na estrutura ou função do concepto. Informações sobre a conduta a ser tomada no caso de exposições a teratogênicos durante a gestação são imprescindíveis. Com essa finalidade, em 2001, foi implantado o Serviço de Informações sobre Agentes Teratogênicos da Bahia (SIAT-BA), localizado no Serviço de Genética Médica do Hospital Universitário Professor Edgard Santos da Universidade Federal da Bahia. O objetivo é descrever as características do atendimento do SIAT-BA nos seus primeiros quatro anos de funcionamento. As consultas foram realizadas através do contato via telefone, fax ou e-mail, entre março de 2001 e maio de 2005. Nesse período, o SIAT-BA recebeu 408 consultas, totalizando 1.091 motivos. A maioria das consultas foi realizada por gestantes e profissionais de saúde. Produtos para cabelo, chás e misoprostol foram os agentes mais investigados. O pequeno número de consultas (1/dia) indica a necessidade de maior divulgação do serviço e da conscientização da população sobre os riscos oferecidos pelos diversos agentes.

Teratogênios; Anormalidades Congênitas; Gravidez; Serviços de Informação 


\section{Colaboradores}

M. B. Toralles e C. Alves participaram da análise dos dados, orientação, correção e edição do trabalho. B. M. C. Trindade, L. C. Fadul, C. F. Peixoto Junior e M. A. C. C. Santana colaboraram no levantamento de dados, revisão da literatura e redação do artigo.

\section{Agradecimentos}

Esse trabalho foi parcialmente financiado pela Fundação de Amparo à Pesquisa do Estado da Bahia (FAPESB).

\section{Referências}

1. Shepard TH. Agents that cause birth defects. Yonsei Med J 1995; 36:393-6.

2. Hansen WF, Peacock AE, Yankowitz J. Safe prescribing practices in pregnancy and lactation. J Midwifery Womens Health 2002; 47:409-21.

3. Wannmacher L. Fármacos em gestação e lactação. In: Fuchs FD, Wannmacher L, organizadores. Farmacologia clínica: fundamentos da terapêutica racional. Rio de Janeiro: Editora Guanabara Koogan; 2004. p. 936-41.

4. Opitz JM. Tópicos recentes em genética clínica. Ribeirão Preto: Sociedade Brasileira de Genética; 1982.

5. Schüller-Faccini L, Schvartzman L, Cecchin C. Teratogênese humana e o SIAT. In: Sanseverino MT Spritzer D, Schüller-Faccini L, organizadores. Manual de teratogênese. Porto Alegre: Editora da Universidade; 2001. p. 11-7.

6. European Network of Teratology Information Services. ENTIS. http://www.entis-org.com (acessado em 10/Jan/2008).

7. Clementi M, Di Gianantonio E, Ornoy A. Teratology information services in Europe and their contribution to the prevention of congenital anomalies. Community Genet 2002; 5:8-12.
8. Leen-Mitchell M, Martinez L, Gallegos S, Robertson J, Carey JC. Mini-review: history of organized teratology information services in North America. Teratology 2000; 61:314-7.

9. Schuler L, Sanseverino MT, Clavijo HA, AshtonProlla PB, Karen PF, Costa FS, et al. Preliminary report on the first Brazilian teratogen information service (SIAT). Rev Bras Genét 1993; 16:1085-95.

10. Rocha RS, Costa FS, Eleutério FJC, Moreira JLC, Guimarães JA, Schüler-Faccini L, et al. Sistemas de informação sobre agentes teratogênicos no Brasil e no mundo. Femina 2006; 35:309-15.

11. Schüler L, Pastuszak A, Sanseverino TV, Orioli IM, Brunoni D, Ashton-Prolla P, et al. Pregnancy outcome after exposure to misoprostol in Brazil: a prospective, controlled study. Reprod Toxicol 1999; 13:147-51.

12. Orioli IM, Castilla EE. Epidemiological assessment of misoprostol teratogenicity. BJOG 2000; 107: 519-23.

13. Schüller-Faccini L, Leite JCL, Sanseverino MTV, Peres RM. Avaliação de teratógenos potenciais na população brasileira. Ciênc Saúde Coletiva 2002; 7:65-71.

Recebido em 15/Fev/2007

Versão final reapresentada em 11/Fev/2008

Aprovado em 26/Jun/2008 